IJMMS 27:11 (2001) 701-706

PII. S0161171201007190

http://ijmms.hindawi.com

(C) Hindawi Publishing Corp.

\title{
AN EXTENSION OF A THEOREM OF SAHAB, KHAN, AND SESSA
}

\author{
A. R. KHAN and N. HUSSAIN
}

(Received 20 March 2001)

\begin{abstract}
A fixed point theorem of Fisher and Sessa is generalized to locally convex spaces and the new result is applied to extend a recent theorem on invariant approximation of Sahab, Khan, and Sessa.
\end{abstract}

2000 Mathematics Subject Classification. 41A65, 46A03, 47H10.

1. Introduction and terminology. In 1988, Mukherjee and Verma [10] obtained the following generalization of a theorem of Fisher and Sessa [4].

THEOREM 1.1. Let $T$ and I be two weakly commuting mappings of a closed convex subset $C$ of a Banach space $X$ into itself satisfying the inequality

$$
\|T x-T y\| \leq a\|I x-I y\|+(1-a) \max \{\|T x-I x\|,\|T y-I y\|\},
$$

for all $x, y \in C$, where $a \in(0,1)$. If I is affine and nonexpansive in $C$ and if $T(C) \subseteq I(C)$, then $T$ and $I$ have a unique common fixed point in $C$.

In this note, we first prove that Theorem 1.1 can appreciably be extended to the setup of a Hausdorff locally convex space. An application of new result is presented to best approximation theory; our work extends earlier results of Brosowski [3], Sahab et al. [12], Singh [14] and many others.

In the sequel, $(E, \tau)$ will be a Hausdorff locally convex topological vector space. A family $\left\{p_{\alpha}: \alpha \in I\right\}$ of seminorms defined on $E$ is said to be an associated family of seminorms for $\tau$ if the family $\{\gamma U: \gamma>0\}$, where $U=\bigcap_{i=1}^{n} U_{\alpha_{i}}$ and $U_{\alpha_{i}}=\{x$ : $\left.p_{\alpha_{i}}(x)<1\right\}$, forms a base of neighbourhoods of zero for $\tau$. A family $\left\{p_{\alpha}: \alpha \in I\right\}$ of seminorms defined on $E$ is called an augmented associated family for $\tau$ if $\left\{p_{\alpha}: \alpha \in I\right\}$ is an associated family with the property that the seminorm $\max \left\{p_{\alpha}, p_{\beta}\right\} \in\left\{p_{\alpha}: \alpha \in I\right\}$ for any $\alpha, \beta \in I$. The associated and augmented associated families of seminorms will be denoted by $A(\tau)$ and $A^{*}(\tau)$, respectively. It is well known that given a locally convex space $(E, \tau)$, there always exists a family $\left\{p_{\alpha}: \alpha \in I\right\}$ of seminorms defined on $E$ such that $\left\{p_{\alpha}: \alpha \in I\right\}=A^{*}(\tau)$ (see [9, page 203]).

The following construction will be crucial. Suppose that $M$ is a $\tau$-bounded subset of $E$. For this set $M$ we can select a number $\lambda_{\alpha}>0$ for each $\alpha \in I$ such that $M \subset \lambda_{\alpha} U_{\alpha}$ where $U_{\alpha}=\left\{x: p_{\alpha}(x) \leq 1\right\}$. Clearly $B=\bigcap_{\alpha} \lambda_{\alpha} U_{\alpha}$ is $\tau$-bounded, $\tau$-closed, absolutely convex and contains $M$. The linear span $E_{B}$ of $B$ in $E$ is $\bigcup_{n=1}^{\infty} n B$. The Minkowski functional of $B$ is a norm $\|\cdot\|_{B}$ on $E_{B}$. Thus $\left(E_{B},\|\cdot\|_{B}\right)$ is a normed space with $B$ as its closed unit ball and $\sup _{\alpha} p_{\alpha}\left(x / \lambda_{\alpha}\right)=\|x\|_{B}$ for each $x \in E_{B}$. 
Following Sessa [13], we say, two selfmaps $I$ and $T$ of a locally convex space $(E, \tau)$ are weakly commuting if and only if

$$
p_{\alpha}(I T x-T I x) \leq p_{\alpha}(I x-T x)
$$

for each $x \in E$ and $p_{\alpha} \in A^{*}(\tau)$. Clearly, commuting maps are weakly commuting but not conversely in general (see [10,13]). A mapping $T: E \rightarrow E$ is said to be nonexpansive on $E$ if $p_{\alpha}(T x-T y) \leq p_{\alpha}(x-y)$ for all $x, y$ in $E$ and $p_{\alpha} \in A^{*}(\tau)$. The set of fixed points of $T$ on $E$ is denoted by $F(T)$. If $u \in E, M \subseteq E$, then for $0<a \leq 1$, we define the set $D_{a}$ of best $(M, a)$-approximants to $u$ as follows:

$$
D_{a}=\left\{y \in M: a p_{\alpha}(y-u)=d_{p_{\alpha}}(u, M), \forall p_{\alpha} \in A^{*}(\tau)\right\}
$$

where

$$
d_{p_{\alpha}}(u, M)=\inf \left\{p_{\alpha}(x-u): x \in M\right\} .
$$

Let $D$ denote the set of best approximations to $u$. For $a=1$, our definition reduces to the set $D$ of best $M$-approximants to $u$. A mapping $T: M \rightarrow E$ is called demiclosed at 0 if whenever $\left\{x_{n}\right\}$ converges weakly to $x$ and $\left\{T x_{n}\right\}$ converges to 0 , we have $T x=0$.

\section{Results}

LEMMA 2.1. Let $T$ and $I$ be weakly commuting selfmaps of a $\tau$-bounded subset $M$ of a Hausdorff locally convex space $(E, \tau)$. Then $T$ and I are weakly commuting on $M$ with respect to $\|\cdot\|_{B}$.

Proof. By hypothesis for any $x \in M$,

$$
p_{\alpha}(I T x-T I x) \leq p_{\alpha}(I x-T x), \text { for each } p_{\alpha} \in A^{*}(\tau) .
$$

Taking supremum on both sides, we get

$$
\begin{gathered}
\sup _{\alpha} p_{\alpha}\left(\frac{I T x-T I x}{\lambda_{\alpha}}\right) \leq \sup _{\alpha} p_{\alpha}\left(\frac{I x-T x}{\lambda_{\alpha}}\right), \\
\|I T x-T I x\|_{B} \leq\|I x-T x\|_{B} \quad \text { as desired. }
\end{gathered}
$$

Note that if $I$ is nonexpansive on a $\tau$-bounded subset $M$ of $E$, then $I$ is also nonexpansive with respect to $\|\cdot\|_{B}$ (cf. $\left.[8,15]\right)$.

We use a technique of Tarafdar [15] to obtain the following common fixed point theorem which generalizes Theorem 1.1 and the main result of Fisher and Sessa [4].

THEOREM 2.2. Let $M$ be a nonempty $\tau$-bounded, $\tau$-complete, and convex subset of a Hausdorff locally convex space $(E, \tau)$ and $T, I$ two weakly commuting selfmaps of $M$ satisfying the inequality

$$
p_{\alpha}(T x-T y) \leq a p_{\alpha}(I x-I y)+(1-a) \max \left\{p_{\alpha}(T x-I x), p_{\alpha}(T y-I y)\right\},
$$

for all $x, y \in M$ and for all $p_{\alpha} \in A^{*}(\tau)$ and for some $a \in(0,1)$. If $I$ is affine and nonexpansive on $M$ and $T(M) \subseteq I(M)$, then $T$ and $I$ have $a$ unique common fixed point. 
Proof. Since $M$ is $\tau$-complete, it follows that $\left(E_{B},\|\cdot\|_{B}\right)$, is a Banach space and $M$ is complete in it. By Lemma $2.1, T$ and $I$ are $\|\cdot\|_{B}$-weakly commuting maps of $M$. From (2.3) we obtain for $x, y \in M$,

$$
\begin{aligned}
\sup _{\alpha} p_{\alpha}\left(\frac{T x-T y}{\lambda_{\alpha}}\right) \leq & a \sup _{\alpha} p_{\alpha}\left(\frac{I x-I y}{\lambda_{\alpha}}\right) \\
& +(I-a) \max \left\{\sup _{\alpha} p_{\alpha}\left(\frac{T x-I x}{\lambda_{\alpha}}\right), \sup _{\alpha} p_{\alpha}\left(\frac{T y-I y}{\lambda_{\alpha}}\right)\right\} .
\end{aligned}
$$

Thus

$$
\|T x-T y\|_{B} \leq a\|I x-I y\|_{B}+(1-a) \max \left\{\|T x-I x\|_{B},\|T y-I y\|_{B}\right\} .
$$

It can be shown easily that $I$ is $\|\cdot\|_{B}$-nonexpansive on $M$. A comparison of our hypothesis with that of Theorem 1.1 tells that we can apply Theorem 1.1 to $M$ as a subset of $\left(E_{B},\|\cdot\|_{B}\right)$ to conclude that there exists a unique $a \in M$ such that $a=I a=T a$.

An application of Theorem 2.2 establishes the following result in best approximation theory.

THEOREM 2.3. Let $T$ and I be selfmaps of a Hausdorff locally convex space $(E, \tau)$ and $M$ a subset of $E$ such that $T(\partial M) \subseteq M$, where $\partial M$ denotes boundary of $M$ and $u \in F(T) \cap F(I)$. Suppose that $T$ and I satisfy (2.3) for all $x, y$ in $D_{a}^{\prime}=D_{a} \cup\{u\}$ and $I$ is nonexpansive and affine on $D_{a}$. For each $p_{\alpha} \in A^{*}(\tau)$,

$$
p_{\alpha}(T I x-I T x) \leq \frac{1}{k} p_{\alpha}((k T x+(1-k) q)-I x),
$$

for all $k \in(0,1), x \in D_{a}$ and for some $q \in D_{a}$. If $D_{a}$ is nonempty convex, $q \in F(I)$ and $I\left(D_{a}\right)=D_{a}$, then $I$ and $T$ have a common fixed point in $D_{a}$ provided one of the following conditions holds:

(i) $D_{a}$ is $\tau$-compact.

(ii) $D_{a}$ is weakly compact in $(E, \tau), I$ is weakly continuous and $I-T$ is demiclosed at 0 .

Proof. Let $y \in D_{a}$. Then $I y \in D_{a}$, since $I\left(D_{a}\right)=D_{a}$. Further, if $y \in \partial M$, then $I y \in M$ for $T(\partial M) \subseteq M$. From (2.3), it follows that for each $p_{\alpha} \in A^{*}(\tau)$,

$$
\begin{aligned}
p_{\alpha}(T y-u) & =p_{\alpha}(T y-T u) \\
& \leq a p_{\alpha}(I y-I u)+(1-a) \max \left\{p_{\alpha}(T y-I y), p_{\alpha}(T u-I u)\right\} \\
& \leq a p_{\alpha}(I y-u)+(1-a)\left(p_{\alpha}(T y-u)+p_{\alpha}(I y-u)\right) .
\end{aligned}
$$

So we have, $a p_{\alpha}(T y-u) \leq p_{\alpha}(I y-u)$ for all $p_{\alpha} \in A^{*}(\tau)$. Hence $T y \in D_{a}$ which implies that $T$ maps $D_{a}$ into itself.

Let $\left\{k_{n}\right\}$ be a monotonically nondecreasing sequence of real numbers such that $0<k_{n}<1$ and $\limsup k_{n}=1$. Define for each $n \in \mathbb{N}$, a mapping $T_{n}: D_{a} \rightarrow D_{a}$ by

$$
T_{n}(x)=k_{n} T x+\left(1-k_{n}\right) q .
$$

It is possible to define such a mapping $T_{n}$ for each $n \in \mathbb{N}$, since $D_{a}$ is convex and 
$q \in D_{a}$. The map $I$ is affine so we have

$$
T_{n} I x=k_{n} T I x+\left(1-k_{n}\right) q, \quad I T_{n} x=k_{n} I T x+\left(1-k_{n}\right) q .
$$

From (2.6), it follows that

$$
\begin{aligned}
p_{\alpha}\left(T_{n} I x-I T_{n} x\right) & =k_{n} p_{\alpha}(T I x-I T x) \\
& \leq k_{n}\left(\left(\frac{1}{k_{n}}\right) p_{\alpha}\left(k_{n} T x+\left(1-k_{n}\right) q\right)-I x\right) \\
& =p_{\alpha}\left(T_{n} x-I x\right), \quad \forall x \in D_{a}, p_{\alpha} \in A^{*}(\tau) .
\end{aligned}
$$

Thus $I$ and $T_{n}$ are weakly commuting on $D_{a}$ for each $n$ and $T_{n}\left(D_{a}\right) \subseteq D_{a}=I\left(D_{a}\right)$. For all $x, y \in D_{a}, p_{\alpha} \in A^{*}(\tau)$ and for all $j \geq n$, ( $n$ fixed), we obtain from (2.3),

$$
\begin{aligned}
p_{\alpha}\left(T_{n} x-T_{n} y\right)= & k_{n} p_{\alpha}(T x-T y) \leq k_{j} p_{\alpha}(T x-T y) \\
\leq & p_{\alpha}(T x-T y) \\
\leq & a p_{\alpha}(I x-I y)+(1-a) \max \left\{p_{\alpha}(T x-I x), p_{\alpha}(T y-I y)\right\} \\
\leq & a p_{\alpha}(I x-I y)+(1-a) \max \left\{p_{\alpha}\left(T x-T_{n} x\right)+p_{\alpha}\left(T_{n} x-I x\right),\right. \\
& \left.p_{\alpha}\left(T y-T_{n} y\right)+p_{\alpha}\left(T_{n} y-I y\right)\right\} \\
\leq & a p_{\alpha}(I x-I y)+(1-a) \max \left\{\left(1-k_{n}\right) p_{\alpha}(T x-q)+p_{\alpha}\left(T_{n} x-I x\right),\right. \\
& \left.\left(1-k_{n}\right) p_{\alpha}(T y-q)+p_{\alpha}\left(T_{n} y-I y\right)\right\} .
\end{aligned}
$$

Hence for all $j \geq n$, we have

$$
\begin{aligned}
& p_{\alpha}\left(T_{n} x-T_{n} y\right) \leq a p_{\alpha}(I x-I y) \\
&+(1-a) \max \left\{\left(1-k_{j}\right) p_{\alpha}(T x-q)+p_{\alpha}\left(T_{n} x-I x\right),\right. \\
&\left.\quad\left(1-k_{j}\right) p_{\alpha}(T y-q)+p_{\alpha}\left(T_{n} y-I y\right)\right\} .
\end{aligned}
$$

As $\lim k_{j}=1$, from (2.12), for every $n \in \mathbb{N}$, we have

$$
\begin{aligned}
p_{\alpha}\left(T_{n} x-T_{n} y\right)= & \lim _{j} p_{\alpha}\left(T_{n} x-T_{n} y\right) \\
\leq & \lim _{j}\left\{a p_{\alpha}(I x-I y)+(1-a)\right. \\
& \times \max \left\{\left(1-k_{j}\right) p_{\alpha}(T x-q)+p_{\alpha}\left(T_{n} x-I x\right),\right. \\
& \left.\left.\left(1-k_{j}\right) p_{\alpha}(T y-q)+p_{\alpha}\left(T_{n} y-I y\right)\right\}\right\} .
\end{aligned}
$$

This implies that for every $n \in \mathbb{N}$,

$$
p_{\alpha}\left(T_{n} x-T_{n} y\right) \leq a p_{\alpha}(I x-I y)+(1-a) \max \left\{p_{\alpha}\left(T_{n} x-I x\right), p_{\alpha}\left(T_{n} y-I y\right)\right\},
$$

for all $x, y \in D_{a}$ and for all $p_{\alpha} \in A^{*}(\tau)$.

(i) $D_{a}$ being $\tau$-compact is $\tau$-bounded and $\tau$-complete. Thus by Theorem 2.2, for every $n \in \mathbb{N}, T_{n}$ and $I$ have unique common fixed point $x_{n}$ in $D_{a}$. Now the $\tau$-compactness 
of $D_{a}$ ensures that $\left\{x_{n}\right\}$ has a convergent subsequence $\left\{x_{n_{j}}\right\}$ which converges to a point $x_{o} \in D_{a}$. Since

$$
x_{n_{j}}=T_{n_{j}} x_{n_{j}}=k_{n_{j}} T x_{n_{j}}+\left(1-k_{n_{j}}\right) q
$$

and $T$ is continuous, so we have, as $j \rightarrow \infty, T x_{0}=x_{0}$. The continuity of $I$ implies that

$$
I x_{o}=I\left(\lim _{j} x_{n_{j}}\right)=\lim _{j} I\left(x_{n_{j}}\right)=\lim _{j} x_{n_{j}}=x_{0} .
$$

(ii) Weakly compact sets in $(E, \tau)$ are $\tau$-bounded and $\tau$-complete so again by Theorem 2.2, $T_{n}$ and $I$ have a common fixed point $x_{n}$ in $D_{a}$ for each $n$. The set $D_{a}$ is weakly compact so there is a subsequence $\left\{x_{j}\right\}$ of $\left\{x_{n}\right\}$ converging weakly to some $y \in D_{a}$. The map $I$ being weakly continuous gives that $I y=y$. Now

$$
x_{j}=I\left(x_{j}\right)=T_{j}\left(x_{j}\right)=k_{j} T x_{j}+\left(1-k_{j}\right) q
$$

implies that $I x_{j}-T x_{j}=\left(1-k_{j}\right)\left[q-T x_{j}\right] \rightarrow 0$ as $j \rightarrow \infty$. The demiclosedness of $I-T$ at 0 implies that $(I-T)(y)=0$. Hence $I y=T y=y$.

EXAMPLE 2.4 (cf. MR.89h:54030). Let $M=[1, \infty)$ and $d$ be the absolute value metric on $M$. Define $f$ and $g$ on $M$ by $f x=1+x, g(x)=1+2 x$. As $d(f g x, g f x)=1 \leq x=$ $d(f x, g x)$ for all $x$ in $M$ so $f$ and $g$ are weakly commuting but evidently there exists no sequence $\left\{x_{n}\right\}$ in $M$ for which the condition of compatibility is satisfied ( $f$ and $g$ are compatible (see [6]) if $d\left(f g x_{n}, g f x_{n}\right) \rightarrow 0$, as $n \rightarrow \infty$, for any sequence $\left\{x_{n}\right\}$ in $M$ satisfying $\left.\lim _{n} f x_{n}=\lim _{n} g x_{n}=t \in M\right)$.

REMARKS 2.5. (i) In the light of Example 2.4, the classes of weakly commuting and compatible maps are different and so the statement "weakly commuting maps are compatible" on page 977 in [6] is not valid. Hence Theorem 2.3 cannot be implied by Theorem 5 of Pathak et al. [11] even in Banach space setting.

(ii) Commuting maps satisfy (2.6) so Theorem 2.3(i) is a proper generalization of the main results of Sahab et al. [12] and Singh [14].

ACKNOWLEDGEMENT. A. R. Khan gratefully acknowledges the support provided by the King Fahd University of Petroleum and Minerals during this research.

\section{REFERENCES}

[1] M. A. Al-Thagafi, Common fixed points and best approximation, J. Approx. Theory 85 (1996), no. 3, 318-323. MR 97f:47051. Zbl 858.41022.

[2] R. Baskaran and P. V. Subrahmanyam, Common fixed points in closed balls, Atti Sem. Mat. Fis. Univ. Modena 36 (1988), no. 1, 1-5. MR 89k:47088. Zbl 656.54031.

[3] B. Brosowski, Fixpunktsätze in der Approximationstheorie, Mathematica (Cluj) 11 (34) (1969), 195-220. MR 43\#3712. Zbl 207.45502.

[4] B. Fisher and S. Sessa, On a fixed point theorem of Greguš, Int. J. Math. Math. Sci. 9 (1986), no. 1, 23-28. MR 87g:47102. Zbl 597.47036.

[5] M. Greguš, Jr., A fixed point theorem in Banach space, Boll. Un. Mat. Ital. A (5) 17 (1980), no. 1, 193-198. MR 81c:47058. Zbl 538.47035.

[6] G. Jungck, Common fixed points for commuting and compatible maps on compacta, Proc. Amer. Math. Soc. 103 (1988), no. 3, 977-983. MR 89h:54030. Zbl 661.54043. 
[7] G. Jungck and S. Sessa, Fixed point theorems in best approximation theory, Math. Japon. 42 (1995), no. 2, 249-252. MR 96f:47112. Zbl 834.54026.

[8] A. R. Khan, N. Hussain, and L. A. Khan, A note on Kakutani type fixed point theorems, Int. J. Math. Math. Sci. 24 (2000), no. 4, 231-235. MR 2001f:47096. Zbl 0968.41016.

[9] Gottfried Köthe, Topological Vector Spaces. I, Die Grundlehren der mathematischen Wissenschaften, vol. 159, Springer-Verlag, New York, 1969. MR 40\#1750. Zbl 179.17001.

[10] R. N. Mukherjee and V. Verma, A note on a fixed point theorem of Greguš, Math. Japon. 33 (1988), no. 5, 745-749. MR 90g:47102. Zbl 655.47047.

[11] H. K. Pathak, Y. J. Cho, and S. M. Kang, An application of fixed point theorems in best approximation theory, Int. J. Math. Math. Sci. 21 (1998), no. 3, 467-470. MR 99d:47055. Zbl 909.47044.

[12] S. A. Sahab, M. S. Khan, and S. Sessa, A result in best approximation theory, J. Approx. Theory 55 (1988), no. 3, 349-351. MR 90a:41032. Zbl 676.41031.

[13] S. Sessa, On a weak commutativity condition of mappings in fixed point considerations, Publ. Inst. Math. (Beograd) (N.S.) 32(46) (1982), 149-153. MR 85f:54107. Zbl 523.54030.

[14] S. P. Singh, An application of a fixed-point theorem to approximation theory, J. Approx. Theory 25 (1979), no. 1, 89-90. MR 80G:41026. Zbl 0399.41032.

[15] E. Tarafdar, Some fixed-point theorems on locally convex linear topological spaces, Bull. Austral. Math. Soc. 13 (1975), no. 2, 241-254. MR 53\#8979. Zbl 318.47032.

A. R. Khan: Department of Mathematical Sciences, King Fahd University of PetroLEUM AND MiNERALS, DHAHRAN 31261, SAUdi ARABIA

E-mail address: arahim@kfupm.edu.sa

N. Hussain: Center for Advanced Studies in Pure AND Applied Mathematics, BAHAUDDIN ZAKARIYA UNIVERSITY, MULTAN 60800, PAKISTAN

E-mail address: mnawab2000@yahoo.com 


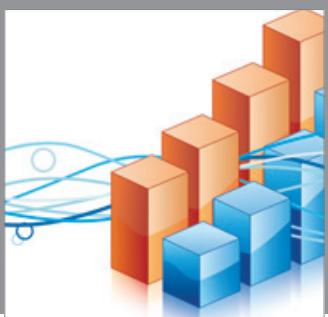

Advances in

Operations Research

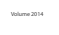

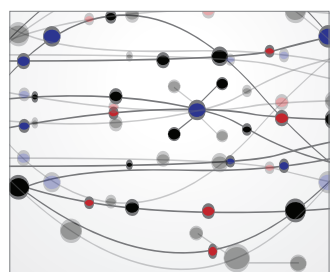

\section{The Scientific} World Journal
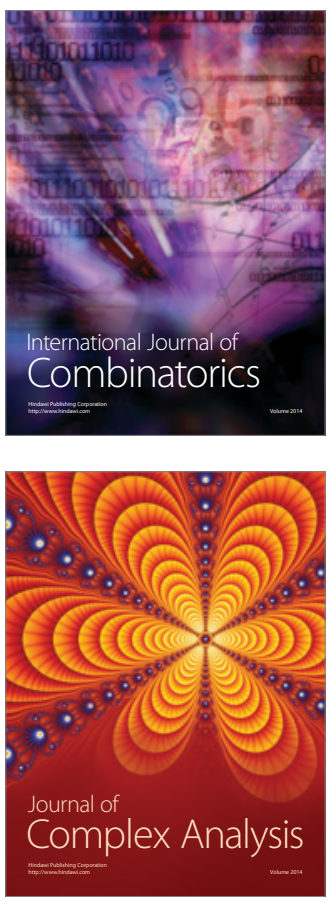

International Journal of

Mathematics and

Mathematical

Sciences
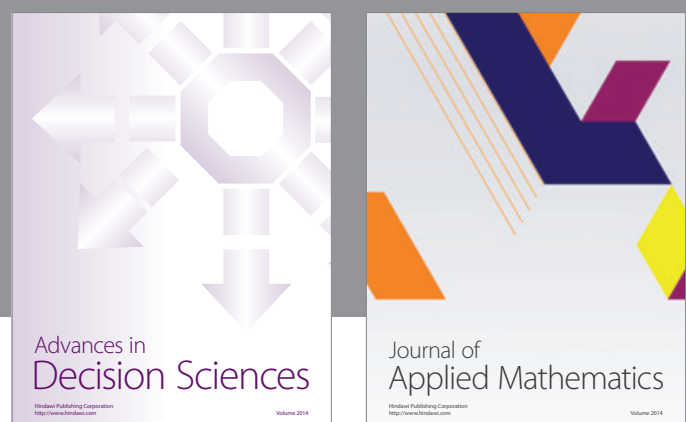

Journal of

Applied Mathematics
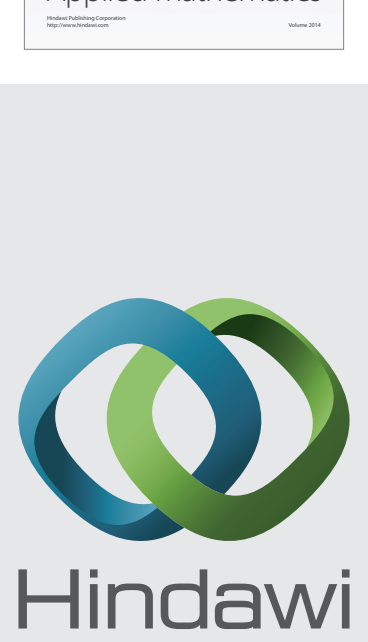

Submit your manuscripts at http://www.hindawi.com
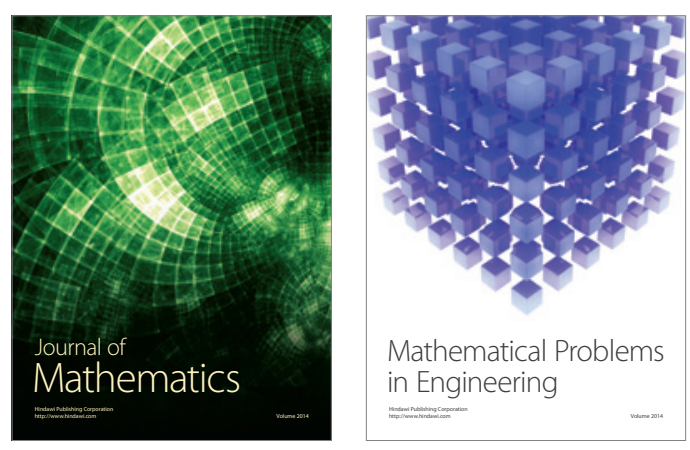

Mathematical Problems in Engineering
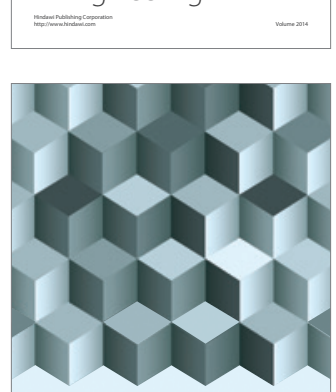

Journal of

Function Spaces
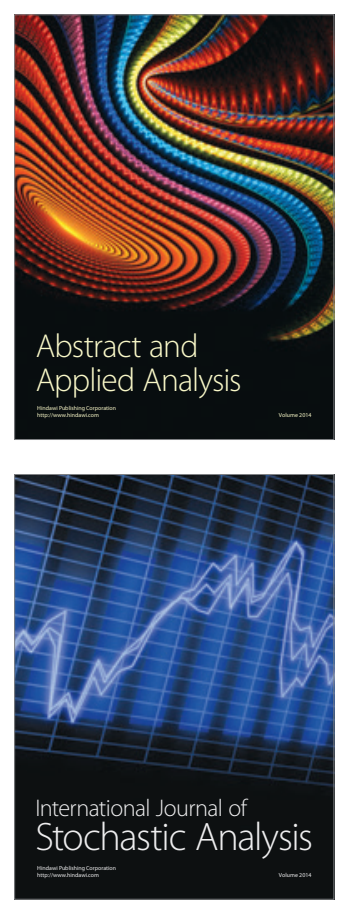

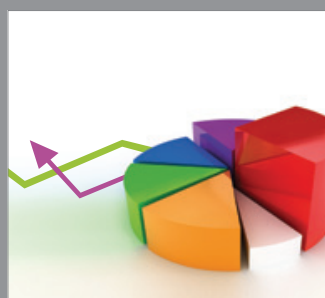

ournal of

Probability and Statistics

Promensencen
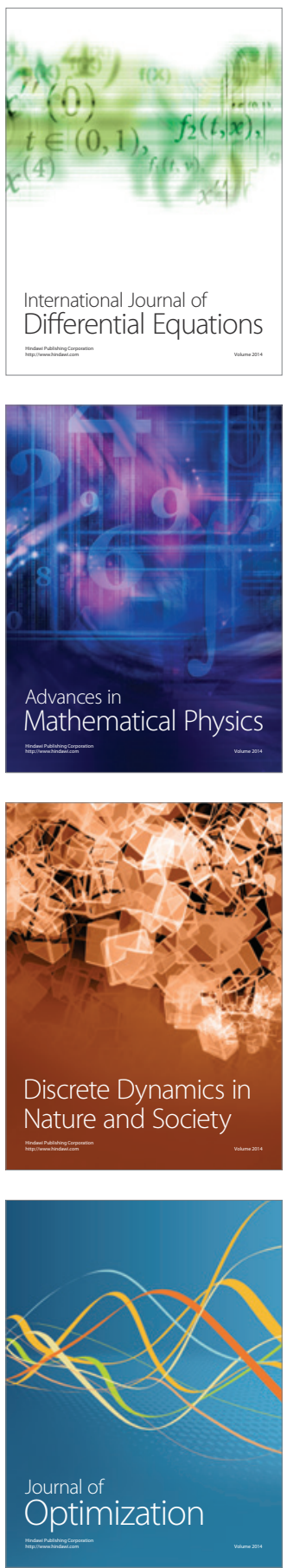